OPEN ACCESS

Edited by:

Béla Birkás,

University of Pécs, Hungary

Reviewed by:

X. T. Wang,

The Chinese University of

Hong Kong, China

Joyce Lok Yin Kwan,

The Education University of

Hong Kong, Hong Kong

${ }^{*}$ Correspondence: Hui Jing Lu

huijing.lu@polyu.edu.hk

Specialty section: This article was submitted to Evolutionary Psychology, a section of the journal

Frontiers in Psychology

Received: 01 May 2020 Accepted: 17 June 2020

Published: 28 July 2020

Citation:

Guo S, Lu HJ, Zhu N and Chang L (2020) Meta-Analysis of Direct and Indirect Effects of Father Absence on Menarcheal Timing.

Front. Psychol. 11:1641.

doi: 10.3389/fpsyg.2020.01641

\section{Meta-Analysis of Direct and Indirect Effects of Father Absence on Menarcheal Timing}

\author{
Shaolingyun Guo ${ }^{1}$, Hui Jing Lu ${ }^{1 *}$, Nan $\mathrm{Zhu}^{2}$ and Lei Chang ${ }^{2}$ \\ ${ }^{1}$ Department of Applied Social Sciences, The Hong Kong Polytechnic University, Kowloon, Hong Kong, ${ }^{2}$ Department of \\ Psychology, University of Macau, Taipa, Macau
}

Despite extensive evidence of the association between father absence and early onset of menarche, whether father absence directly accelerates the onset of menarche or the association is mediated by other negative family psychosocial processes remains unclear. Reliable theories on the basis of which father absence has been investigated also vary. Within the life history $(\mathrm{LH})$ theoretical framework, we conducted a meta-analysis of studies that investigated father absence, menarcheal timing, and various family disturbances that cause stress in children. We tested the hypothesis that father absence exerts a direct effect on menarcheal timing and an indirect effect on menarcheal timing mediated by integrated childhood stress. Quantitative synthesis using a two-stage meta-analytic structural equation modeling approach was applied to test our hypothesis. Based on seven research articles $(N=4,619)$ that include at least one form of family stressor as well as father absence and menarcheal timing, integrated childhood stress emerged as a robust mediator of the association between father absence and early menarcheal timing, and the total effect of father absence on menarcheal timing had reduced in size after accounting for the mediating effect of childhood stress. The findings emphasize the importance of a father figure in regulating a child's LH, including menarcheal timing.

Keywords: meta-analysis, father absence, childhood stress, menarche, mediation

\section{INTRODUCTION}

Early childhood experiences deeply influence female reproductive development, including the timing of puberty, which is generally indicated by the age of menarche (Karapanou and Papadimitriou, 2010). Age at menarche depends on the interaction between genes and the environment (DiVall and Radovick, 2008); moreover, the effects of genetic factors do not preclude environmental and psychosocial factors affecting pubertal timing (Graber et al., 1995; Chasiotis et al., 1998; Ellis et al., 1999). To explain the potential effects of environmental factors on menarcheal timing, researchers have proposed different theories, including the paternal investment and resource scarcity hypothesis (Draper and Harpending, 1982; Belsky et al., 1991), male shortage model (Hoier, 2003), and different aspects of the life history (LH) theoretical framework (Draper and Harpending, 1982; Belsky et al., 1991; Chisholm, 1993; Ellis, 2004; Belsky, 2012). However, the explanations as well as the effects of father absence on early onset of menarche reported in these studies are inconsistent or equivocal. Some studies have demonstrated that father absence is associated with 
early onset of menarche (e.g., Moffitt et al., 1992; Quinlan, 2003; Webster et al., 2014), whereas other studies have shown that father absence did not affect menarcheal timing (Campbell and Udry, 1995; D’Onofrio et al., 2006; Sohn, 2017). In addition, whether father absence directly affects menarcheal timing or the effect is mediated by other mechanisms remains unclear. The present meta-analysis adopted the LH framework to examine empirical research that explored factors accelerating the onset of menarche and investigated the indirect effects of father absence on menarcheal timing.

\section{Menarcheal Timing and the Life History Strategy}

Menarcheal timing is one of the few physical LH traits that have been investigated in human studies (Del Giudice, 2020). Conceptualized within the LH theoretical framework, menarcheal timing assumes to be a good indicator of the fastslow LH continuum. Early menarcheal timing is associated with the faster end of the continuum that trades growth, development, and learning for early maturation and reproduction. The faster LH strategy results in rapid development, relatively high mating effort, and raising numerous offspring with relatively low parental investment. By contrast, late menarcheal timing is associated with the slow LH strategy, which is characterized by delayed reproduction because of the allocation of bioenergy to somatic development and learning and relatively high parental investment in raising and training high-quality offspring (Ellis et al., 2009; Del Giudice et al., 2015; Ellis and Del Giudice, 2019). The adopted LH trade-off strategy and menarcheal timing vary in response to environmental factors and are determined by environmental constraints. Of the two major environmental constraints that determine animal LH, namely, resource constraint or food shortage and safety constraint or extrinsic risk, food shortage has become less relevant in contemporary human life because of sufficient food supplies that exceed the survival threshold (Chang and Lu, 2018). The diminished role of food shortage over the economic growth is also evident in the stable secular trend of menarcheal age shifting over the past century from the late teenage years to the early teenage years in recent times (Soliman et al., 2014; Chang and Lu, 2018).

The second environmental constraint, namely, safety constraint or extrinsic risk, is particularly relevant in shaping human LH strategies (Ellis et al., 2009). Major safety threats in evolution include predation, disease, and intraspecific violence. These extrinsic risks or safety threats cause mortality and morbidity that are beyond an individual's survival efforts. Known as environmental harshness and unpredictability, the frequency of and variation in extrinsic risks are associated with fast $\mathrm{LH}$ strategies (Ellis et al., 2009). In response to environmental harshness and unpredictability, fast strategists who grow rapidly and mature early are more likely to escape death and disability in the post-reproductive period. Moreover, fast strategists who produce many offspring are more likely to hedge against juvenile mortality and achieve higher fitness than individuals who exhibit high parental investment, which is ineffective in preventing juvenile mortality. By contrast, slow LH strategists prevail in safe and predictable environments. They invest time and energy in their own physical and mental development (acquiring knowledge and skills) as well as in the development of their offspring; consequently, generations of slow strategists are more competitive in certain ecological contexts (i.e., where high skills are favored) than fast strategists who allocate higher amounts of energy to mating than to learning and development or parenting and training their offspring.

\section{Direct and Indirect Effects of Father Absence}

As a potential evolved adaptive response, fast-slow $\mathrm{LH}$ tradeoff strategies continue to regulate development and behavior in response to current environmental constraints (Pepper and Nettle, 2017). According to the literature, contemporary proxies of environmental harshness and unpredictability that have been extensively investigated include low familial socioeconomic status (SES; Belsky et al., 2012), employment and residential changes (Doom et al., 2016; Zuo et al., 2018), unsafe neighborhood and unpredictable life events (Chang et al., 2019), familial chaos (Lu and Chang, 2019), harsh parenting (Mell et al., 2018), and parental absence (Chang and Lu, 2018). Among these microenvironmental proxies, father absence has particularly been implicated in inducing early menarche (Belsky et al., 1991; Ellis, 2004) and other fast LH manifestations (Ellis et al., 2003; Chang and Lu, 2018). Father absence represents environmental harshness and unpredictability because the presence of a father is an essential part of human evolution (Marlowe, 2000; Lu et al., 2015). Paternal care is evidenced in humans by the father assuming the role of a helper at the nest. Paternal and biparental care of the offspring represents one of many interrelated slow LH strategies, including pair bonding, monogamy, and gender equality development (Zhu and Chang, 2019). In terms of both direct parental care and indirect parental investment in the form of provisioning, a father is essential in supporting slow LH strategies and development in human children (Geary, 2000). Father absence causes disruptions in the otherwise stable microenvironment of a child and leads to fast LH manifestations such as early menarche. Concealed ovulation to prolong mate guarding, employment of the father as a helper at the nest, and selecting a suitable mate in terms of paternal value ( $\mathrm{Lu}$ et al., 2015) are evolutionary events that can also be viewed as advancements of female interest in the long-standing conflict of interests between the sexes. This perspective suggests that father absence should particularly upset daughters' LH and cause early menarche. The aforementioned prediction is consistent with the literature that associates father absence with menarche and girls' LH.

In addition to the direct effect on menarche and other fast LH manifestations, father absence affects child LH indirectly by contributing to familial perturbation and stress, which result in fast LH manifestations including the early onset of menarche (Belsky et al., 1991, 2012). The indirect effect of father absence was proposed within the psychosocial acceleration model that focused on how a harsh family environment accelerates children's 
reproductive and LH schedules (Belsky et al., 1991). A harsh family environment includes situations such as father absence, marital conflict, divorce or separation, coercive or abusive parenting, and insufficient or inconsistent economic conditions. Any one of these factors may cause perturbations to other components of the family system and create cascades of harsh and stressful experiences for the child. For example, parents experiencing marital difficulties or economic hardships may exhibit insensitivity, rejection, or inconsistency in child-rearing, whereas stable marital and economic conditions are associated with supportive and consistent parenting as well as secure child attachment. Psychosocial stresses resulting from harsh parenting and other family disturbances were reported to accelerate LH including menarcheal timing (Belsky et al., 1991). According to the psychosocial acceleration model (Belsky et al., 1991), father absence represents one example of possible family disturbances, and children experience stress from harsh family environments, which may accelerate LH and cause early onset of menarche.

\section{Present Study}

The present study examined the indirect effect of father absence on menarche mediated through integrated childhood stress. A meta-analysis was used to examine statistical robustness of the overall trends by integrating the potential effect across studies. Previous meta-analyses or systematic review only focused on the total effect of father absence (Webster et al., 2014; Sohn, 2017), but these studies did not include other potential variables that may mediate the relation between father absence and menarcheal timing. The present study applied structural equation modeling in a meta-mediation analysis to identify and explicate the process underlying the observed relationship between father absence (independent variable) and menarcheal age (dependent variable) through a mediating process or intervening variable involving stresses experienced by the child (Figure 1). Full mediation refers to when the regression coefficient for path $c^{\prime}$ is not significantly different from zero; and partial mediation is said to occur if path c' has decreased in size relative to the coefficient for path c but remains significant (Baron and Kenny, 1986). The aforementioned stresses include family conflict, low SES, negative family relationship, and other child-perceived stresses. A two-stage structural equation modeling (TSSEM) approach [see (Cheung and Chan, 2009)] was adopted to identify, examine, and quantify the indirect effect of father absence on menarcheal timing mediated by integrated childhood stress.

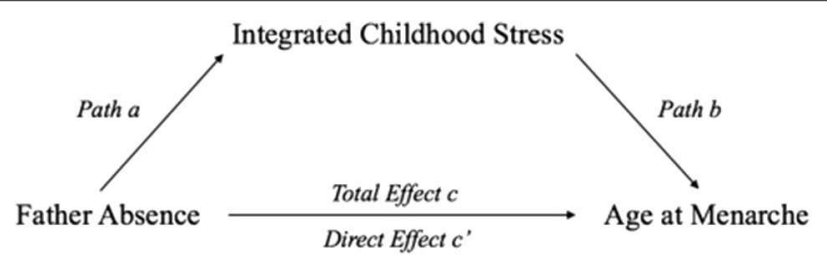

FIGURE 1 | Single mediator model. The total effect is presented as path c, and the direct effect is presented as path c'.

\section{METHODS}

\section{Search Strategy and Study Selection}

The present meta-analysis included studies obtained after relevant searches in the following electronic databases: PsycINFO, PubMed, and Google Scholar. Relevant publications were systematically searched using their titles, keywords, and abstracts. The keywords used were "menarche" and "stress," and the related terms used were "childhood stress," "hardship," "adversity," "family conflict," "violence," "childhood abuse," "maltreatment," "mistreatment," "low family income," "financial difficulty," and "low SES." Furthermore, the keyword "father absence" and related terms, including "father absence," "paternal absence," and "parental absence," were used. Additional relevant publications were identified by manually searching in reference lists of reviewed articles.

Studies were selected if they reported (1) the daughters' age or timing of menarche, either concurrently or retrospectively, which was measured using a single item of age or an item embedded in a multi-item measurement of pubertal timing; (2) father absence, missing father figure, or timing and duration of father absence; (3) childhood stress, including perceived childhood or early life stress, stressful family environment, childhood hardship or adversity, early life depression, family conflict, low SES, or childhood abuse; (4) regression coefficients or bivariate correlations between father absence and menarcheal age, between childhood stress and menarcheal age, and between father absence and childhood stress; (5) the mechanism between father absence and menarcheal age through a mediating process or intervening variable involving childhood stresses experienced after father absence. Figure 2 illustrates the flowchart of study selection. Where there were queries regarding the data, authors were contacted for further information; if authors either did not respond or did not provide additional information, we excluded those studies. Table 1 presents the characteristics of the selected studies, including the author(s), year of publication, study sample size, average age or age range of participants, measure of father absence, measure of childhood stress, menarcheal age, and study design of each study.

The validity of each included study was assessed using the STrengthening the Reporting of OBservational studies in Epidemiology (STROBE). All the included studies were evaluated based on the following criteria: the informativeness of the title and abstract, justifiability of the research rationale, rigorousness of the research design, credibility of data sources, accuracy of data analyses, clarity of finding presentation, and relevance of discussion (Vandenbroucke et al., 2014). 14 items adopted from the STROBE were relevant to the aforementioned criteria and assigned a score of 0 or 1 . Out of a total score of 14, all the included studies had scores of at least 11 (Table 2), indicating that the validity of the studies based on the STROBE criteria was adequate (Elm et al., 2007; Adams et al., 2018).

\section{Data Analysis}

A meta-analytic structural equation modeling (SEM; MASEM) approach was used to estimate the mediating effects involving 


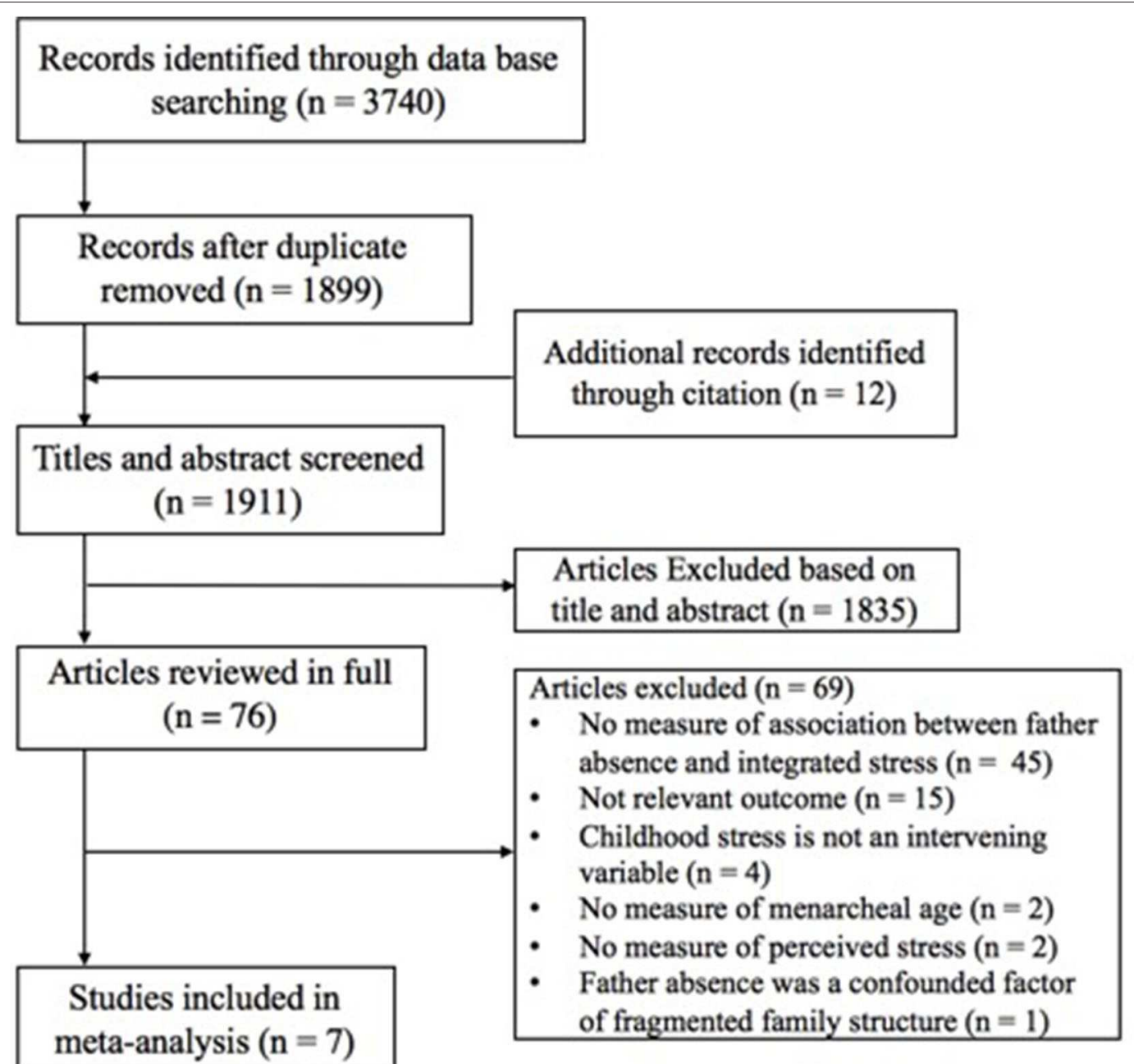

FIGURE 2 | Flow diagram depicting the article selection process.

father absence, integrated stress, and menarche onset age. The MASEM approach was combined with SEM and meta-analysis by synthesizing correlations or covariance matrices between $\mathrm{X}$ (independent variable), $\mathrm{M}$ (mediator), and $\mathrm{Y}$ (dependent variable) across studies and fitting SEMs on the pooled correlation or covariance matrix (Landis, 2013). Meta-analyses were performed using the TSSEM approach adopted from the metaSEM package of $\mathrm{R}$ (Cheung and Chan, 2005). In the first stage of the TSSEM approach, a pooled correlation matrix was created using the independent variable, mediator, and outcome variable weighted with the sample size of each study (Cheung and Chan, 2009). That is, in order to conduct TSSEM analyses, regression coefficients or bivariate correlations between $\mathrm{X}$ (father absence), M (childhood stress), and Y (menarcheal age or menarcheal timing) were extracted from each relevant study. Corresponding study sample sizes were also extracted. If the correlation matrices were not homogeneous, a pooled correlation matrix was estimated and produced (Cheung, 2013). The second stage of the TSSEM approach involved treating the pooled matrix as the observed correlation matrix and fitting a meta-structural mediational model to test the fit of model to the data (see Cheung, 2013). The pooled correlation matrix was used to fit the stage two structural model, with the asymptotic covariance matrix of the pooled correlation matrix as the weight matrix generated by the weighted least squares (WLS) method (Cheung, 2013). As samples, study design and effect sizes were expected to be different across studies; a random-effects model was adopted with the assumption that population correlation matrices may vary. The random-effects model is generally considered to be more conservative in a meta-analysis in the presence of heterogeneity. Unstandardized regression coefficients and their corresponding standard errors extracted from the stage two TSSEM results were then used to conduct a Sobel test to determine the significance of the indirect pathway from father absence on menarcheal age through childhood stress (Sobel, 1982; Preacher and Hayes, 2008). Supplementary R code to this article is outlined in Appendix A. 


\begin{tabular}{|c|c|c|c|c|c|c|c|c|}
\hline References & Sample & $N$ & Age & Father absence & $\begin{array}{l}\text { Types of } \\
\text { childhood stress }\end{array}$ & Measures of stressful experiences & $\begin{array}{l}\text { Puberty } \\
\text { timing/menarche }\end{array}$ & $\begin{array}{l}\text { Study } \\
\text { design }\end{array}$ \\
\hline $\begin{array}{l}\text { Chang and Lu } \\
\text { (2018) }\end{array}$ & $\begin{array}{l}\text { A community sample was taken } \\
\text { from a randomly selected rural } \\
\text { county in Henan Province. }\end{array}$ & 57 & $\begin{array}{l}\text { The average } \\
\text { ages of the } \\
\text { left-behind } \\
\text { and non-left- } \\
\text { behind } \\
\text { children were } \\
10.78 \text { and } \\
10.69 \text {. }\end{array}$ & $\begin{array}{l}\text { Father absence: estimated the } \\
\text { amount of time that one or both } \\
\text { parents spent away from the } \\
\text { child. }\end{array}$ & $\begin{array}{l}\text { Child-perceived } \\
\text { stress }\end{array}$ & $\begin{array}{l}12 \text { items from the literature (e.g., } \\
\text { Goodman, 1997) to measure this } \\
\text { construct (e.g., When I was growing } \\
\text { up, they [my parents or people I lived } \\
\text { with] fought a lot; they were not } \\
\text { around). The items were rated on a } \\
\text { 6-point scale. }\end{array}$ & $\begin{array}{l}\text { The age of } \\
\text { menarche }\end{array}$ & Cohort \\
\hline $\begin{array}{l}\text { Culpin et al. } \\
\text { (2014) }\end{array}$ & $\begin{array}{l}\text { The sample comprised } \\
\text { participants from the Avon } \\
\text { Longitudinal Study of Parents } \\
\text { and children resident in the } \\
\text { former Avon Health Authority in } \\
\text { southwest England. }\end{array}$ & 2,750 & $\begin{array}{l}8 \text { years old up } \\
\text { to age } 17 \\
\text { years. }\end{array}$ & $\begin{array}{l}\text { A three-level categorical variable } \\
\text { to examine potential differential } \\
\text { effects of timing of father } \\
\text { absence. }\end{array}$ & $\begin{array}{l}\text { Early life } \\
\text { socioeconomic } \\
\text { stressor }\end{array}$ & $\begin{array}{l}\text { Range of indicators of family } \\
\text { socioeconomic disadvantage as } \\
\text { major financial problems. }\end{array}$ & $\begin{array}{l}\text { Age at onset of } \\
\text { menarche }\end{array}$ & Cohort \\
\hline $\begin{array}{l}\text { Ellis et al. } \\
\text { (1999) }\end{array}$ & $\begin{array}{l}\text { Participating families were initially } \\
\text { recruited from three geographical } \\
\text { areas (Nashville and Knoxville, } \\
\text { Tennessee, and Bloomington, } \\
\text { Indiana). }\end{array}$ & 281 & $\begin{array}{l}\text { Aged 12-13 } \\
\text { years old. }\end{array}$ & $\begin{array}{l}\text { Father absence and } \\
\text { single-parent status: amount of } \\
\text { time the father spent taking care } \\
\text { of child, on a 4-point scale } \\
\text { ranging from } 1 \text { (brief care) to } 4 \\
\text { (major care). }\end{array}$ & $\begin{array}{l}\text { Severity of conflict } \\
\text { in the parental } \\
\text { dyad }\end{array}$ & $\begin{array}{l}\text { Severity of conflict within the parental } \\
\text { dyad [this included the biological } \\
\text { mother and the male partner (either } \\
\text { husband or boyfriend) living in the } \\
\text { home] on a 5-point scale ranging } \\
\text { from } 1 \text { (rarely even shout) to } 5 \\
\text { (physical fights, more than once). }\end{array}$ & $\begin{array}{l}\text { Pubertal } \\
\text { development } \\
\text { scores were } \\
\text { converted into } \\
\text { pubertal timing } \\
\text { scores. }\end{array}$ & Cohort \\
\hline $\begin{array}{l}\text { James et al. } \\
\text { (2012) }\end{array}$ & $\begin{array}{l}\text { Participants were enrolled in a } \\
\text { longitudinal study of adolescence } \\
\text { in the United States. }\end{array}$ & 240 & $\begin{array}{l}\text { Mean age }= \\
11.86 \text { years; } \\
S D=0.56\end{array}$ & $\begin{array}{l}\text { Father absence was reported as } \\
0=\text { father present; } 1=\text { father } \\
\text { absence. }\end{array}$ & $\begin{array}{l}\text { Negative family } \\
\text { relationship }\end{array}$ & $\begin{array}{l}\text { Mothers' reports of negative family } \\
\text { relationships were assessed via } \\
\text { Conflict Subscale of the Family } \\
\text { Environment Scale (FES), General } \\
\text { Functioning Subscale of the Family } \\
\text { Assessment Device (FAD), and The } \\
\text { Conflict Behavior Questionnaire } \\
\text { (CBQ). }\end{array}$ & Age of menarche & Cohort \\
\hline $\begin{array}{l}\text { Moffitt et al. } \\
\text { (1992) }\end{array}$ & $\begin{array}{l}\text { Subjects were adolescent girls } \\
\text { involved in the Dunedin (New } \\
\text { Zealand) Multidisciplinary Health } \\
\text { and Development Study. }\end{array}$ & 326 & NA & $\begin{array}{l}\text { Mother completed one rating of } \\
\text { the amount of time the father } \\
\text { spent taking care of child, } \\
\text { ranging from } 1 \text { (brief care) to } 4 \\
\text { (major care). }\end{array}$ & Family conflict & $\begin{array}{l}\text { Mothers were asked to recall each } \\
\text { era and to answer this question: "All } \\
\text { families have conflicts, parents and } \\
\text { kids. What kinds of family strife and } \\
\text { violence was your child exposed to } \\
\text { during this time" (e.g., shouting, } \\
\text { physical fights, } \\
\text { pushing - parent-parent or } \\
\text { parent-child)?" }\end{array}$ & Age of menarche & Cohort \\
\hline $\begin{array}{l}\text { Richardson } \\
\text { et al. (2018) }\end{array}$ & $\begin{array}{l}\text { Data were collected from a } \\
\text { University in the Southeastern } \\
\text { United States that serves } \\
\text { students from predominantly } \\
\text { rural backgrounds. }\end{array}$ & 342 & $\begin{array}{l}\text { Aged } 18-49 \\
\text { years with a } \\
\text { mean age of } \\
20.7 \text { years. }\end{array}$ & $\begin{array}{l}\text { Indicators of exposure to the } \\
\text { fragmented family structure } \\
\text { asking participants to endorse } \\
\text { when they experienced before } \\
\text { menarche. }\end{array}$ & $\begin{array}{l}\text { Socioeconomic } \\
\text { status (SES) }\end{array}$ & $\begin{array}{l}\text { SES was indexed with indicators } \\
\text { assessing mother's education, } \\
\text { father's education, and childhood } \\
\text { family income. }\end{array}$ & Age at menarche & $\begin{array}{l}\text { Cross- } \\
\text { sectional }\end{array}$ \\
\hline $\begin{array}{l}\text { Vigil and Geary } \\
\text { (2006) }\end{array}$ & $\begin{array}{l}\text { The participants were women } \\
\text { from Albuquerque, New Mexico, } \\
\text { and suburban towns in } \\
\text { mid-Missouri. }\end{array}$ & 623 & $\begin{array}{l}\text { Ages } 18-56 \\
\text { years, } M= \\
26.9 \text { years, } \\
S D=8.5\end{array}$ & $\begin{array}{l}\text { Father involvement was } \\
\text { measured by the amount of time } \\
\text { spent with father measured on a } \\
5 \text {-point scale, from } 1 \text { (never) to } 5 \\
\text { (always). }\end{array}$ & $\begin{array}{l}\text { Parent-child } \\
\text { conflict }\end{array}$ & $\begin{array}{l}\text { A family conflict item that assessed } \\
\text { the degree to which participants } \\
\text { argued and fought with their parents } \\
\text { during childhood (measured on a } \\
\text { 5-point scale). }\end{array}$ & Age of menarche & $\begin{array}{l}\text { Cross- } \\
\text { sectional }\end{array}$ \\
\hline
\end{tabular}

Subjects were adolescent girls involved in the Dunedin (New Zealand) Multidisciplinary Health and Development Study. and suburban towns in (always). 
TABLE 2 | Quality assessments.

\begin{tabular}{|c|c|c|c|c|c|c|c|}
\hline References & $\begin{array}{l}\text { Chang and } \\
\text { Lu (2018) }\end{array}$ & $\begin{array}{l}\text { Culpin et al. } \\
(2014)\end{array}$ & $\begin{array}{l}\text { Ellis et al. } \\
(1999)\end{array}$ & $\begin{array}{l}\text { James et al. } \\
(2012)\end{array}$ & $\begin{array}{l}\text { Moffitt et al. } \\
(1992)\end{array}$ & $\begin{array}{l}\text { Richardson } \\
\text { et al. (2018) }\end{array}$ & $\begin{array}{l}\text { Vigil and } \\
\text { Geary (2006) }\end{array}$ \\
\hline \multicolumn{8}{|l|}{ Title and abstract } \\
\hline $\begin{array}{l}\text { Include abstract, an informative and } \\
\text { balanced summary. }\end{array}$ & 1 & 1 & 1 & 1 & 1 & 1 & 1 \\
\hline \multicolumn{8}{|l|}{ Introduction } \\
\hline $\begin{array}{l}\text { Explain the scientific background, } \\
\text { objective, and rationale. }\end{array}$ & 1 & 1 & 1 & 1 & 1 & 1 & 1 \\
\hline \multicolumn{8}{|l|}{ Methods } \\
\hline Present key elements of study design. & 1 & 1 & 1 & 1 & 1 & 1 & 1 \\
\hline $\begin{array}{l}\text { Describe the setting, locations, and } \\
\text { relevant dates and characteristics of } \\
\text { study participants. }\end{array}$ & 1 & 1 & 1 & 1 & 1 & 1 & 1 \\
\hline $\begin{array}{l}\text { Give the eligibility criteria and the } \\
\text { sources and methods of selection of } \\
\text { participants. }\end{array}$ & 0 & 1 & 1 & 1 & 0 & 0 & 0 \\
\hline $\begin{array}{l}\text { Clearly define all outcomes, } \\
\text { exposures, predictors, potential } \\
\text { confounders, and effect modifiers. }\end{array}$ & 1 & 1 & 1 & 1 & 1 & 1 & 1 \\
\hline $\begin{array}{l}\text { Give sources of data and details of } \\
\text { methods of assessment. }\end{array}$ & 1 & 1 & 1 & 1 & 1 & 1 & 1 \\
\hline $\begin{array}{l}\text { Explain how quantitative variables } \\
\text { were handled in the analyses. }\end{array}$ & 1 & 1 & 1 & 1 & 1 & 1 & 1 \\
\hline $\begin{array}{l}\text { Statistically appropriate/acceptable } \\
\text { methods of data analysis used. }\end{array}$ & 1 & 1 & 0 & 1 & 0 & 1 & 0 \\
\hline \multicolumn{8}{|l|}{ Results } \\
\hline $\begin{array}{l}\text { Give unadjusted estimates and/or } \\
\text { confounder-adjusted estimates and } \\
\text { their precision. }\end{array}$ & 1 & 1 & 1 & 1 & 1 & 1 & 1 \\
\hline $\begin{array}{l}\text { Give a cautious overall interpretation } \\
\text { of results. }\end{array}$ & 1 & 1 & 1 & 1 & 1 & 1 & 1 \\
\hline \multicolumn{8}{|l|}{ Discussion } \\
\hline $\begin{array}{l}\text { Summarize key results with reference } \\
\text { to study objectives. }\end{array}$ & 1 & 1 & 1 & 1 & 1 & 1 & 1 \\
\hline Discuss limitations of the study. & 1 & 1 & 1 & 1 & 0 & 1 & 1 \\
\hline $\begin{array}{l}\text { Discuss the generalizability of the } \\
\text { study results. }\end{array}$ & 1 & 0 & 1 & 1 & 1 & 1 & 1 \\
\hline
\end{tabular}

\section{RESULTS}

\section{Study Flow and Characteristics}

In the database search, 3,740 candidate studies were identified; 1,841 of these studies were duplicates. After additional articles from citation lists were identified, 1,911 studies were obtained. However, after duplicate removal, 1,835 studies were excluded on the basis of titles and abstracts. Thus, 76 studies were available for full-test evaluation. Of these 76 studies, seven included a measure of a childhood stress and sufficient information to calculate bivariate correlations (Figure 2). The characteristics of the seven included articles are shown in Table 1. Among the included seven studies, childhood stress was measured as family or parental conflict $(n=3)$, socioeconomic stress $(n=2)$, negative family relationship $(n=1)$, and perceived childhood stress index $(n=$ 1 ). The study sample size ranged from 57 to 2,750 participants. The proposed mechanisms of childhood stresses are summarized in Table 3.

\section{Two-Stage Structural Equation Modeling Results With Mediation Effect}

The correlation matrix was extracted and synthesized using data from the seven included studies for the first stage of the TSSEM approach. The value of the $\mathrm{Q}$ statistic, which is a measure of the homogeneity of effect sizes, was significant [Q $(18)=144.59, p<0.001]$, thus indicating that notable variation was present across the selected studies. Because of the large sample size and high heterogeneity across multiple studies, random-effects models were used for analysis in stages 1 and 2 of the TSSEM approach. Correlation matrices were extracted and synthesized from the seven included studies for the first stage of the TSSEM. The total pooled sample size was 4,619 . Table 4 presents the pooled correlation coefficients for $\mathrm{X}$ (father absence), M (integrated childhood stress), and $\mathrm{Y}$ (age at menarche), showing that all three correlations are highly significant. 
TABLE 3 | Proposed mechanisms of father absence on early menarche through integrated childhood stress.

\begin{tabular}{|c|c|c|c|c|c|c|}
\hline $\begin{array}{l}\text { Chang and Lu } \\
\text { (2018) }\end{array}$ & $\begin{array}{l}\text { Culpin et al. } \\
(2014)\end{array}$ & Ellis et al. (1999) & $\begin{array}{l}\text { James et al. } \\
(2012)\end{array}$ & $\begin{array}{l}\text { Moffitt et al. } \\
\text { (1992) }\end{array}$ & $\begin{array}{l}\text { Richardson et al. } \\
\text { (2018) }\end{array}$ & Vigil and Geary (2006) \\
\hline $\begin{array}{l}\text { Separate measure of } \\
\text { paternal absence on } \\
\text { pubertal timing (age } \\
\text { of menarche for girls), } \\
\text { father absence was } \\
\text { associated with } \\
\text { increased childhood } \\
\text { perceived stress (lack } \\
\text { of paternal care) that } \\
\text { leads to accelerated } \\
\text { pubertal timing. }\end{array}$ & $\begin{array}{l}\text { Some of the } \\
\text { effects of } \\
\text { father absence } \\
\text { on age at } \\
\text { menarche may } \\
\text { be mediated } \\
\text { through } \\
\text { exposure to } \\
\text { financial } \\
\text { problems. }\end{array}$ & $\begin{array}{l}\text { Father effects on daughters' } \\
\text { pubertal timing should } \\
\text { involve more than just } \\
\text { father-absent effects; that is, } \\
\text { quality of paternal } \\
\text { investment and family life } \\
\text { stress (greater severity of } \\
\text { conflict in the parental dyad) } \\
\text { should predict daughters' } \\
\text { pubertal timing even within } \\
\text { father-present homes. }\end{array}$ & $\begin{array}{l}\text { Within the } \\
\text { model: an } \\
\text { indirect pathway } \\
\text { of father } \\
\text { absence-lower } \\
\text { quality family } \\
\text { relationships- } \\
\text { greater } \\
\text { age-adjusted } \\
\text { pubertal } \\
\text { maturation. }\end{array}$ & $\begin{array}{l}\text { Within the model } \\
\text { of contextual } \\
\text { stress on girls' age } \\
\text { at menarche } \\
\text { whereby family } \\
\text { conflict mediates } \\
\text { the relationship } \\
\text { between father } \\
\text { absence and age } \\
\text { of menarche. }\end{array}$ & $\begin{array}{l}\text { Parental resources and } \\
\text { status (socioeconomic } \\
\text { status is traditionally } \\
\text { indexed with indicators } \\
\text { of paternal resources) } \\
\text { could indirectly affect } \\
\text { environmental/family } \\
\text { structure effects (e.g., } \\
\text { father absence) on age } \\
\text { of menarche (see } \\
\text { two-part SEM paths). }\end{array}$ & $\begin{array}{l}\text { Investment in social status } \\
\text { (financial assistance as } \\
\text { proxies for participants' } \\
\text { social status) may serve } \\
\text { as a cue that mediates the } \\
\text { present-day relation } \\
\text { between background } \\
\text { parental characteristics } \\
\text { (father absence) and } \\
\text { reproductive development } \\
\text { (including the age of } \\
\text { menarche). }\end{array}$ \\
\hline
\end{tabular}

Clarifications are given in parentheses.

TABLE 4 | Pooled correlation coefficients $(k=7)$ for $X$ (father absence), $M$ (integrated childhood stress), and $Y$ (age at menarche) produced in two-stage structural equation modeling (TSSEM) stage one random-effects model.

\begin{tabular}{lccc}
\hline & Father absence & $\begin{array}{c}\text { Integrated } \\
\text { childhood } \\
\text { stress }\end{array}$ & $\begin{array}{c}\text { Age at } \\
\text { menarche }\end{array}$ \\
\hline Father absence & 1 & & \\
Integrated childhood stress & $0.12(0.04)^{\star \star \star}$ & 1 & 1 \\
Age at menarche & $0.13(0.03)^{\star \star \star}$ & $0.29(0.05)^{\star \star \star}$ & 1 \\
\hline
\end{tabular}

Standard errors are displayed in parentheses; ${ }^{* \star \star} p<0.001$.

A stage 2 analysis using the TSSEM approach was conducted on the pooled correlation matrix as an observed covariance matrix to fit the meta-SEMs by applying the weighted least square estimation method. Figure 3 displays the path diagram of the synthesized meta-SEM results employing TSSEM stage 2 random-effects models. Although the regression coefficient for path c' remained significant, it became reduced in size compared to the value of path c ( $\beta=0.10, p=0.005$ for path $c^{\prime} ; \beta=0.13$, $p<0.001$ for path $c)$, which is an indication of partial mediation (Baron and Kenny, 1986). The Sobel test that used correlation estimates between father absence and childhood stress as well as between childhood stress and menarcheal age and their respective standard errors (SEs) showed that the perceived childhood stress significantly mediated the effects of father absence on menarcheal age $(Z=2.64, \mathrm{SE}=0.01, p=0.008)$. This result is consistent with several key findings reported in the literature on early childhood stress, demonstrating that childhood stress is critical in influencing early menarche (e.g., Belsky et al., 1991; Moffitt et al., 1992; Nettle et al., 2012; Cabeza de Baca and Ellis, 2017).

Generally, the findings supported the role of childhood stress as a mediator, regardless of heterogeneity in measures of childhood stress. Based on the meta-mediation model (Figure 3), father absence was associated with a higher level of childhood stress $(\beta=0.12, p<0.001)$, and a higher level of childhood stress was associated with younger menarcheal age $(\beta=0.28$,

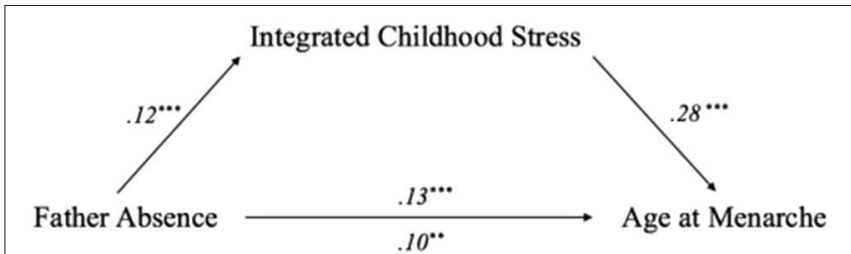

FIGURE 3 | Path analysis diagram depicting two-stage structural equation modeling (TSSEM) stage two random-effects model. The integrated childhood stress partially mediated the causal relationship between father absence and menarcheal age; values are path coefficients. Notes: ${ }^{\star \star} p<0.01, p^{\star \star \star}<0.001$.

$p<0.001)$. Although these associations were small to moderate in terms of pooling effect sizes, the findings provide valuable insight into the role of childhood stress in pubertal development. In addition, despite differences among survey questionnaires, study samples, and methods used in the selected studies, findings from all the studies generally demonstrated that early childhood stress was a significant mediator of the effects of father absence on early onset of menarche. The meta-mediation synthesis provided the evidence suggesting that childhood stress was an underlying mechanism that affected the strength of the main association between father absence and menarcheal age in the present study.

\section{DISCUSSION}

The present meta-analysis examined the indirect association between father absence and menarcheal age through the mediation of childhood stress. Compared with extensive evidence that father absence predicts early menarche, no known studies have systematically reviewed and synthesized the mediating effect of childhood stress to account for the father absence effects on early menarche. Previous studies have reported that girls raised in the absence of their fathers are more likely to reach sexual maturation at a younger age (Ellis et al., 2003; Anderson, 2015; Hehman and Salmon, 2018) because the link between father absence and earlier physical 
maturation is a part of the fast LH strategy (Belsky, 2012; Schlomer et al., 2019). In addition to the total effect of father absence on earlier onset of menarche, the present study showed that father absence and associated stressors, such as familial perturbation and harsh family environment, also affected physical development. The association between menarcheal age and father absence mediated by childhood stress had a smaller regression coefficient than the correlation between father absence and menarcheal age before accounting for the intervening variable. In the seven included studies, integrated childhood stress emerged as a robust mediator, regardless of the heterogeneity among studies and the assumption that all the analyzed studies included random samples from diverse populations.

The present study examined the indirect effect of father absence on the pubertal development of daughters through early life stress. Previous studies have mainly focused on the critical role of father absence in influencing the reproductive strategies of their offspring (Draper and Harpending, 1982; Belsky et al., 1991) or emphasized how menarche was accelerated by childhood stresses, including poor parentchild relationships (Paikoff et al., 1993), children's psychological problems (Moffitt et al., 1992), low SES (Mishra et al., 2009; Belsky et al., 2012), and impoverished and unsafe neighborhoods (Clutterbuck et al., 2014). However, the mechanism through which father absence affects menarcheal age has rarely been examined. Father absence may cause a reduction in household income (because of the lack of material contribution by the father), lack of attention to a child, poor parent-child communication (because the mother has to spend most of her time earning a living), and psychological problems in children because of the harsh survival environment. All consequences of father absence may eventually be internalized into perceived stress by girls, which results in early onset of menarche. This study summarized previous findings and applied meta-mediation analysis to support the aforementioned argument.

The mediating effects of perceived stress in the relationship between father absence and early onset of menarche suggest the possible presence of a causal sequence whereby father absence is a risk factor for the initial perception of familial or psychosocial stress by the child and subsequent early onset of menarche. These findings are consistent with the theoretical underpinnings of stressful circumstances that predict earlier reproductive development (Belsky et al., 1991; Ellis et al., 2003; Tither and Ellis, 2008; Buzney and Decaro, 2012). Performing subgroup analyses of various sources of stress affecting menarcheal timing and longitudinal testing for the aforementioned causal sequence are key directions for future research.

This study has several limitations. First, the present metamediation analysis was limited by a relatively small sample size $(n=7)$ because only a limited number of studies have either included the mediation effect or examined the mediation model of father absence or have reported bivariate correlations or regression coefficients between father absence, childhood stress, and menarcheal age. However, the overall effect size of the mediating effects in the included studies was significant according to the results of the Sobel test, suggesting that childhood stress caused by father absence should not be ignored, even if only a few studies have examined the mechanism underlying how father absence affects early onset of menarche. Second, publication bias was possible in the studies used in this meta-analysis, and current findings may overestimate the effects of father absence on accelerated menarcheal age. This may be due to meta-mediation analyses being conducted on post hoc basis and thus only being reported when there is evidence of an observed relationship between father absence and age of menarche. Nonetheless, numerous studies have reported the effect of father absence (Draper and Harpending, 1982; Chisholm, 1996; Hoier, 2003; Quinlan, 2003; Jorm et al., 2004) and childhood stress (Belsky et al., 1991; Moffitt et al., 1992; Kim and Smith, 1998; Rodgers and Rowe, 2000) on menarcheal age, suggesting that the published effect was actually observed and reports of the effect were reliable but needed more careful interpretations. In addition, the collapse of different family perturbations (e.g., family or parental conflict, socioeconomic stress, negative family relationship, and perceived childhood stress index) as the child experienced stress may attenuate the expected mediating results because of the heterogeneity of these family perturbations. However, the mediating effect was still significant, suggesting that the mediation of perceived stress in the father absence-menarche relationship was robust. Third, the heterogeneity of pooled effects across the analyzed studies was high in the present meta-analysis. The high heterogeneity can be partially explained by differences in study design, study quality, population characteristics, and variable measurement (Higgins, 2003). Random-effects models in the TSSEM approach were developed and used in the present study to reduce the heterogeneity effects (Cheung and Chan, 2009). Fourth, it should be noted that the lack of a non-WEIRD representative sample may have inhibited the degree of variation in association between father absence and menarcheal age. The mechanism through which father absence affects pubertal timing in developing ecological context may be different, e.g., direct paternal influence may be of more importance in determining reproductive behaviors than timing in non-WEIRD populations (Sheppard et al., 2014) and/or the cause of father absence may differ between WEIRD and nonWEIRD populations where father absence is more likely to be caused by paternal death in non-WEIRD (Sear et al., 2019). As the number and quality of mediation studies increase, it would be worthwhile for future studies to address population-specific mechanisms of integrated childhood stress. Future reviews can reduce heterogeneity by clustering similar stresses into subgroups for further analysis. Despite the aforementioned limitations, this study is the first to holistically analyze published data to examine the indirect effect of father absence on early menarche through stress caused by the lack of the father's role in the child's development. 


\section{DATA AVAILABILITY STATEMENT}

The original contributions presented in the study are included in the article/Supplementary Material, further inquiries can be directed to the corresponding author/s.

\section{AUTHOR CONTRIBUTIONS}

SG, HL, NZ, and LC conceived of the presented idea. SG, $\mathrm{HL}$, and LC designed methods of data collection and analysis. SG performed data analysis and wrote the first draft of the manuscript. HL, NZ, and LC commented on and revised drafts

\section{REFERENCES}

Adams, A. D., Benner, R. S., Riggs, T. W., and Chescheir, N. C. (2018). Use of the STROBE checklist to evaluate the reporting quality of observational research in obstetrics. Obstet. Gynecol. 132, 507-512. doi: 10.1097/AOG.0000000000002689

Anderson, K. G. (2015). Father absence, childhood stress, and reproductive maturation in South Africa. Hum. Nat. 26, 401-425. doi: 10.1007/s12110-015-9243-6

Baron, R. M., and Kenny, D. A. (1986). The moderator-mediator variable distinction in social psychological research: conceptual, strategic, and statistical considerations. J. Pers. Soc. Psychol. 51, 1173-1182. doi: 10.1037/0022-3514.51.6.1173

Belsky, J. (2012). The development of human reproductive strategies: progress and prospects. Curr. Dir. Psychol. Sci. 21, 310-316. doi: 10.1177/0963721412453588

Belsky, J., Schlomer, G. L., and Ellis, B. J. (2012). Beyond cumulative risk: distinguishing harshness and unpredictability as determinants of parenting and early life history strategy. Dev. Psychol. 48, 662-673. doi: 10.1037/a0024454

Belsky, J., Steinberg, L., and Draper, P. (1991). Childhood experience, interpersonal development, and reproductive strategy: an evolutionary theory of socialization. Child Dev. 62, 647-670. doi: 10.2307/1131166

Buzney, C. D., and Decaro, J. A. (2012). Explanatory models of female pubertal timing: discordances between cultural models of maturation and the recollection and interpretation of personal developmental experiences. Cult. Med. Psychiatry 36, 601-620. doi: 10.1007/s11013-012-9277-8

Cabeza de Baca, T., and Ellis, B. J. (2017). Early stress, parental motivation, and reproductive decision-making: applications of life history theory to parental behavior. Curr. Opin. Psychol. 15, 1-6. doi: 10.1016/j.copsyc.2017.02.005

Campbell, B. C., and Udry, J. R. (1995). Stress and age at menarche of mothers and daughters. J. Biosocial Sci. 27, 127-134. doi: 10.1017/S0021932000022641

Chang, L., and Lu, H. J. (2018). Resource and extrinsic risk in defining fast life histories of rural Chinese left-behind children. Evol. Hum. Behav. 39, 59-66. doi: 10.1016/j.evolhumbehav.2017.10.003

Chang, L., Lu, H. J., Lansford, J. E., Skinner, A. T., Bornstein, M. H., Steinberg, L., et al. (2019). Environmental harshness and unpredictability, life history, and social and academic behavior of adolescents in nine countries. Dev. Psychol. 55, 890-903. doi: 10.1037/dev0000655

Chasiotis, A., Scheffer, D., Restemeier, R., and Keller, H. (1998). Integenerational context discontinuity affects the onset of puberty. Hum. Nat. 9, 321-329. doi: $10.1007 / \mathrm{s} 12110-998-1008-\mathrm{Z}$

Cheung, M. W. (2013). Fixed- and random-effects meta-analytic structural equation modeling: examples and analyses in R. Behav. Res. Methods 46, 29-40. doi: 10.3758/s13428-013-0361-y

Cheung, M. W., and Chan, W. (2009). A two-stage approach to synthesizing covariance matrices in meta-analytic structural equation modeling. Struct. Equation Model. 16, 28-53. doi: 10.1080/10705510802561295

Cheung, M. W. L., and Chan, W. (2005). Meta-analytic structural equation modeling: a two-stage approach. Psychol. Methods 10, 40-64. doi: 10.1037/1082-989X.10.1.40 of the paper. All authors discussed the results and contributed to the final version of the paper.

\section{FUNDING}

This research was supported by a General Research Fund (Project No. 15608415) from the Research Grants Council of Hong Kong.

\section{SUPPLEMENTARY MATERIAL}

The Supplementary Material for this article can be found online at: https://www.frontiersin.org/articles/10.3389/fpsyg. 2020.01641/full\#supplementary-material

Chisholm, J. S. (1993). Death, hope, and sex: life-history theory and the development of reproductive strategies. Curr. Anthropol. 34, 1-46. doi: $10.1086 / 204131$

Chisholm, J. S. (1996). The evolutionary ecology of attachment organization. Hum. Nat. 7, 1-37. doi: 10.1007/BF02733488

Clutterbuck, S., Adams, J., and Nettle, D. (2014). Frequent residential relocations cumulatively accelerate menarcheal timing in a sample of English adolescent girls. J. Biosocial Sci. 47, 188-202. doi: 10.1017/S0021932014000157

Culpin, I., Heron, J., Araya, R., Melotti, R., Lewis, G., and Joinson, C. (2014). Father absence and timing of menarche in adolescent girls from a UK cohort: the mediating role of maternal depression and major financial problems. J. Adolesc. 37, 291-301. doi: 10.1016/j.adolescence.2014.02.003

Del Giudice, M. (2020). Rethinking the fast-slow continuum of individual differences. Evol. Hum. Behav. (in press).

Del Giudice, M., Gangestad, S. W., and Kaplan, H. S. (2015). "Life history theory and evolutionary psychology," in The Handbook of Evolutionary Psychology, Vol. 1, Foundations, 2nd Edn., ed D. M. Buss (New York, NY: Wiley), 88-114.

DiVall, S. A., and Radovick, S. (2008). Pubertal development and menarche. Ann. N. Y. Acad. Sci. 1135, 19-28. doi: 10.1196/annals.1429.026

D'Onofrio, B. M., Turkheimer, E., Emery, R. E., Slutske, W. S., Heath, A. C. Madden, P. A., et al. (2006). A genetically informed study of the processes underlying the association between parental marital instability and offspring adjustment. Dev. Psychol. 42, 486-499. doi: 10.1037/0012-1649.42.3.486

Doom, J. R., Vanzomeren-Dohm, A. A., and Simpson, J. A. (2016). Early unpredictability predicts increased adolescent externalizing behaviors and substance use: a life history perspective. Dev. Psychopathol. 28, 1505-1516. doi: $10.1017 /$ S0954579415001169

Draper, P., and Harpending, H. (1982). Father absence and reproductive strategy: an evolutionary perspective. J. Anthropol. Res. 38, 255-273. doi: 10.1086/jar.38.3.3629848

Ellis, B. J. (2004). Timing of pubertal maturation in girls: an integrated life history approach. Psychol. Bull. 130, 920-958. doi: 10.1037/0033-2909.130.6.920

Ellis, B. J., Bates, J. E., Dodge, K. A., Fergusson, D. M., Horwood, L. J., Pettit, G. S., et al. (2003). Does father absence place daughters at special risk for early sexual activity and teenage pregnancy? Child Dev. 74, 801-821. doi: 10.1111/1467-8624.00569

Ellis, B. J., and Del Giudice, M. (2019). Developmental adaptation to stress: an evolutionary perspective. Ann. Rev. Psychol. 70, 111-139. doi: 10.1146/annurev-psych-122216-011732

Ellis, B. J., Figueredo, A. J., Brumbach, B. H., and Schlomer, G. L. (2009). Fundamental dimensions of environmental risk: the impact of harsh versus unpredictable environments on the evolution and development of life history strategies. Hum. Nat. 20, 204-268. doi: 10.1007/s12110-009-9063-7

Ellis, B. J., McFadyen-Ketchum, S., Dodge, K. A., Pettit, G. S., and Bates, J. E. (1999). Quality of early family relationships and individual differences in the timing of pubertal maturation in girls: a longitudinal test of an evolutionary model. $J$. Pers. Soc. Psychol. 77, 387-401. doi: 10.1037/0022-3514.77.2.387

Elm, E. V., Altman, D. G., Egger, M., Pocock, S. J., Gøtzsche, P. C., and Vandenbroucke, J. P. (2007). The strengthening the reporting 
of observational studies in epidemiology (STROBE) statement: guidelines for reporting observational studies. Prev. Med. 45, 247-251. doi: 10.1016/j.ypmed.2007.08.012

Geary, D. C. (2000). Evolution and proximate expression of human paternal investment. Psychol. Bull. 126, 55-77. doi: 10.1037/0033-2909.126.1.55

Goodman, R. (1997). The strengths and difficulties questionnaire: A research note. J. Child Psychol. Psychiatry 38, 581-586. doi: 10.1111/j.1469-7610.1997.tb01545.x

Graber, J. A., Brooks-Gunn, J., and Warren, M. P. (1995). The antecedents of menarcheal age: heredity, family environment, and stressful life events. Child Dev. 66, 346-359. doi: 10.2307/1131582

Hehman, J. A., and Salmon, C. A. (2018). Sex-specific developmental effects of father absence on casual sexual behavior and life history strategy. Evol. Psychol. Sci. 5, 121-130. doi: 10.1007/s40806-018-0173-5

Higgins, J. P. (2003). Measuring inconsistency in meta-analyses. BMJ 327, 557-560. doi: 10.1136/bmj.327.7414.557

Hoier, S. (2003). Father absence and age at menarche: a test of four evolutionary models. Hum. Nat. 14, 209-233. doi: 10.1007/s12110-003-1004-2

James, J., Ellis, B. J., Schlomer, G. L., and Garber, J. (2012). Sex-specific pathways to early puberty, sexual debut, and sexual risk taking: tests of an integrated evolutionary-developmental model. Dev. Psychol. 48, 687-702. doi: $10.1037 / \mathrm{a} 0026427$

Jorm, A. F., Christensen, H., Rodgers, B., Jacomb, P. A., and Easteal, S. (2004). Association of adverse childhood experiences, age of menarche, and adult reproductive behaviour: does the androgen receptor gene play a role? Am. J. Med. Genet. B 125B, 105-111. doi: 10.1002/ajmg.b.20114

Karapanou, O., and Papadimitriou, A. (2010). Determinants of menarche. Reprod. Biol. Endocrinol. 8:115. doi: 10.1186/1477-7827-8-115

Kim, K., and Smith, P. K. (1998). Childhood stress, behavioural symptoms and mother-daughter pubertal development. J. Adolesc. 21, 231-240. doi: 10.1006/jado.1998.0149

Landis, R. S. (2013). Successfully combining meta-analysis and structural equation modeling: recommendations and strategies. J. Bus. Psychol. 28, 251-261. doi: $10.1007 / \mathrm{s} 10869-013-9285-\mathrm{x}$

Lu, H. J., and Chang, L. (2019). Aggression and risk taking as adaptive implementations of fast life history strategy. Dev. Sci. 22:e12827. doi: $10.1111 /$ desc. 12827

Lu, H. J., Zhu, X. Q., and Chang, L. (2015). Good genes, good providers, and good fathers: economic development involved in how women select a mate. Evol. Behav. Sci. 9, 215-228. doi: 10.1037/ebs0000048

Marlowe, F. (2000). Paternal investment and the human mating system. Behav. Process. 51, 45-61. doi: 10.1016/S0376-6357(00)00118-2

Mell, H., Safra, L., Algan, Y., Baumard, N., and Chevallier, C. (2018). Childhood environmental harshness predicts coordinated health and reproductive strategies: a cross-sectional study of a nationally representative sample from France. Evol. Hum. Behav. 39, 1-8. doi: 10.1016/j.evolhumbehav.2017.08.006

Mishra, G. D., Cooper, R., Tom, S. E., and Kuh, D. (2009). Early life circumstances and their impact on menarche and menopause. Womens Health 5, 175-190. doi: 10.2217/17455057.5.2.175

Moffitt, T., Caspi, A., Belsky, J., and Silva, P. (1992). Childhood experience and the onset of menarche: a test of a sociobiological model. Child Dev. 64, 47-58. doi: $10.2307 / 1130900$

Nettle, D., Frankenhuis, W. E., and Rickard, I. J. (2012). The adaptive basis of psychosocial acceleration: comment on beyond mental health, life history strategies articles. Dev. Psychol. 48, 718-721. doi: 10.1037/a0027507

Paikoff, R. L., Ford, S. C., and Gunn, J. B. (1993). Mother-daughter dyads view the family: associations between divergent perceptions and daughter well-being. $J$. Youth Adolesc. 22, 473-492. doi: 10.1007/BF01537711

Pepper, G. V., and Nettle, D. (2017). The behavioural constellation of deprivation: causes and consequences. Behav. Brain Sci. 40, 1-72. doi: $10.1017 / \mathrm{S} 0140525 \mathrm{X} 1600234 \mathrm{X}$
Preacher, K. J., and Hayes, A. F. (2008). Asymptotic and resampling strategies for assessing and comparing indirect effects in multiple mediator models. Behav. Res. Methods 40, 879-891. doi: 10.3758/BRM.40.3.879

Quinlan, R. J. (2003). Father absence, parental care, and female reproductive development. Evol. Hum. Behav. 24, 376-390. doi: 10.1016/S1090-5138(03)00039-4

Richardson, G. B., Guardia, A. C. L., and Klay, P. M. (2018). Determining the roles of father absence and age at menarche in female psychosocial acceleration. Evol. Hum. Behav. 39, 437-446. doi: 10.1016/j.evolhumbehav.2018.03.009

Rodgers, J. L., and Rowe, D. C. (2000). Genetic Influences on Human Fertility and Sexuality: Theoretical and Empirical Contributions From the Biological and Behavioral Sciences. Boston, MA: Kluwer Academic. doi: 10.1007/978-1-4615-4467-8

Schlomer, G. L., Murray, J., Yates, B., Hair, K., and Vandenbergh, D. J. (2019). Father absence, age at menarche, and sexual behaviors in women: evaluating the genetic confounding hypothesis using the androgen receptor gene. Evol. Behav. Sci. 13, 205-222. doi: 10.1037/ebs0000137

Sear, R., Sheppard, P., and Coall, D. (2019). Cross-cultural evidence does not support universal acceleration of puberty in father-absent households. Philos. Trans. R. Soc. Lond. B. Biol. Sci. 374:20180124. doi: 10.1098/rstb.2018.0124

Sheppard, P., Snopkowski, K., and Sear, R. (2014). Father absence and reproduction-related outcomes in Malaysia, a transitional fertility population. Hum. Nat. 25, 213-234. doi: 10.1007/s12110-014-9195-2

Sobel, M. E. (1982). Asymptotic confidence intervals for indirect effects in structural equation models. Soc. Methodol. 13, 290-312. doi: 10.2307/270723

Sohn, K. (2017). The null relation between father absence and earlier menarche. Hum. Nat. 28, 407-422. doi: 10.1007/s12110-017-9299-6

Soliman, A., De Sanctis, V., and Elalaily, R. (2014). Nutrition and pubertal development. Ind. J. Endocrinol. Metab. 18, S39-S47. doi: $10.4103 / 2230-8210.145073$

Tither, J. M., and Ellis, B. J. (2008). Impact of fathers on daughters age at menarche: a genetically and environmentally controlled sibling study. Dev. Psychol. 44, 1409-1420. doi: 10.1037/a0013065

Vandenbroucke, J. P., Elm, E. V., Altman, D. G., Gøtzsche, P. C., Mulrow, C. D., Pocock, S. J., et al. (2014). Strengthening the reporting of observational studies in epidemiology (STROBE): explanation and elaboration. Int. J. Surg. 12, 1500-1524. doi: 10.1016/j.ijsu.2014.07.014

Vigil, J. M., and Geary, D. C. (2006). Parenting and community background and variation in women's life-history development. J. Family Psychol. 20, 597-604. doi: 10.1037/0893-3200.20.4.597

Webster, G. D., Graber, J. A., Gesselman, A. N., Crosier, B. S., and Schember, T. O. (2014). A life history theory of father absence and menarche: a meta-analysis. Evol. Psychol. 12, 272-294. doi: 10.1177/147470491401200202

Zhu, N., and Chang, L. (2019). Evolved but not fixed: a life history account of gender roles and gender inequality. Front. Psychol. 10:1709. doi: $10.3389 /$ fpsyg.2019.01709

Zuo, S., Huang, N., Cai, P., and Wang, F. (2018). The lure of antagonistic social strategy in unstable socioecological environment: residential mobility facilitates individuals' antisocial behavior. Evol. Hum. Behav. 39, 364-371. doi: 10.1016/j.evolhumbehav.2018.03.002

Conflict of Interest: The authors declare that the research was conducted in the absence of any commercial or financial relationships that could be construed as a potential conflict of interest.

Copyright $\odot 2020 \mathrm{Guo}, \mathrm{Lu}$, Zhu and Chang. This is an open-access article distributed under the terms of the Creative Commons Attribution License (CC BY). The use, distribution or reproduction in other forums is permitted, provided the original author(s) and the copyright owner(s) are credited and that the original publication in this journal is cited, in accordance with accepted academic practice. No use, distribution or reproduction is permitted which does not comply with these terms. 